\title{
Evaluación de un sistema de radio UWB para posicionamiento preciso de vehículos
}

\author{
Antonio R. Jiménez, Jorge Godoy, Fernando Seco \\ Centro de Automática y Robótica, CAR-CSIC/UPM \\ Ctra. Campo Real km 0.2 \\ 28500. Arganda del Rey. Madrid \\ \{antonio.jimenez, jorge.godoy, fernando.seco\}@csic.es
}

\section{Resumen}

En este trabajo presentamos la evaluación de un equipo de balizas de radio Ultra-Wide-Band (UWB) como sistema complementario de posicionamiento de vehículos en entornos con pobre cobertura GPS. La evaluación del sistema UWB usa como referencia verdadera un sistema RTK-GPS con precisión centimétrica. Las pruebas realizadas en las pistas del CAR muestran que el sistema $U W B$ permite realizar mediciones de rango hasta 90 metros de distancia con precisión submétrica en la mayoría de las medidas y con la aparición de pocos outliers. Colocando 6 nodos UWB en el entorno y dos nodos en el techo de un coche, y utilizando un filtro de Kalman extendido, es posible localizar en $2 D$ y en orientación con buena precisión (0.8 m y 10 grados, respectivamente en el $90 \%$ de los casos). El estudio marca las bases para una posible utilización de UWB en otros entornos (subterráneos o parkings) y en labores de cooperación entre vehículos (platooning).

Palabras clave: Localización, medida de rangos, conducción automática, radio UWB, GPS diferencial

\section{Introducción}

Uno de los retos fundamentales en el campo de los vehículos inteligentes es conseguir un sistema de localización con la suficiente precisión, fiabilidad y robustez. La solución más precisa utilizada en exteriores son los sistemas de localización basados en los sistemas globales de navegación por satélite (GNSS por sus siglas en inglés); como las redes GPS y GLONASS, operadas por EE.UU. y Rusia respectivamente. Este tipo de soluciones están limitadas por diversos efectos de interferencia, bloqueo y propagación multitrayecto de las señales provenientes de los satélites, lo cual merma considerablemente su precisión [4].

Se han propuesto diversas estrategias para conseguir localizar en zonas cubiertas, o con poca cobertura GPS, algunas basadas en señales de radio, acústicas, lidar o basadas en visión [5, 3]. De entre

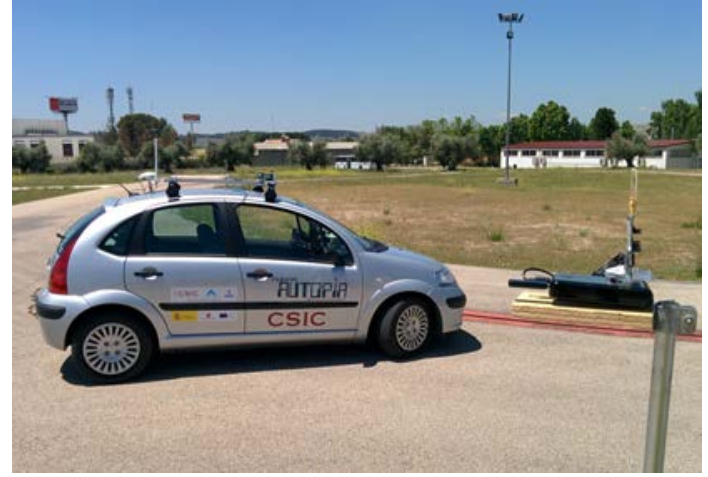

Figura 1: Vehículo del programa Autopia, instrumentado con nodos UWB, utilizado para realizar los test de posicionamiento.

ellas posiblemente la que tiene mayor potencial y que ha tenido una gran repercusión en estos años recientes es la técnica UWB (Ultra-Wide-Band) $[2,7]$. Con ella se consiguen precisiones del orden de 0.2 metros en condiciones LOS (Line-of-Sight).

En este trabajo presentamos la evaluación de un equipo de balizas de radio UWB como sistema complementario de posicionamiento de vehículos en entornos con pobre cobertura GPS. Se presenta el equipamiento utilizado para realizar los tests (sección 2), un estudio de la precisión en la medida de rangos (sección 3), el método de posicionamiento utilizado basado en un filtro de Kalman extendido robustificado (sección 4), el análisis de la precisión obtenida en posicionamiento y orientación (sección 5) y unas conclusiones finales sobre trabajos futuros.

\section{Descripción del equipamiento y del entorno de test}

\subsection{El equipo UWB}

La tecnología UWB o radio impulsiva consiste en emitir pulsos RF de muy corta duración y medir su tiempo de vuelo. Al ser emisiones muy breves utilizan un espectro ancho de frecuencias. Esta emisión espectral está limitada y regulada (FCC) para que no interfiera con otros equipos de medida. La emisión de estos pulsos estrechos tiene la venta- 
ja de que son distinguibles en recepción incluso en condiciones de multicamino, lo cual permite a UWB operar incluso en entornos de interior con múltiples objetos y atravesando paredes.

Existen diferentes productos comerciales UWB tales como Timedomain (www.timedomain.com), Ubisense (www.ubisense.net), Zebra (www.zebra.com), Bespoon (bespoon.com) y Decawave (www.decawave.com). Compañias como DecaWave y BeSpoon usan el estándar de radio impulsiva IEEE 802.15 .4a para definir la capa física que tiene tres rangos de frecuencia: por debajo de $1 \mathrm{GHz}$, entre 3 y $5 \mathrm{GHz}$, y entre 6 y 10 GHz. Por otro lado hay compañías que no utilizan el estándar y usan soluciones propietarias, por ejemplo Time Domain (PulsON -P400) y la serie 7000 de Ubisense. Nosotros en este trabajo vamos a utilizar el kit TREK1000 de Decawave que utiliza el estándar.

El componente base del kit (chip DW1000) permite obtener una precisión en la medida del tiempo de vuelo, usando emisión de ida y vuelta (TWR o Two-Way-Ranging), que redunda en una precisión en rango de $\pm 10 \mathrm{~cm}$. Una distancia máxima de medida de hasta 300 metros es posible en condiciones ideales (sin obstáculos ni reflectores cercanos).

Disponemos para los tests de 8 nodos UWB (Fig.2), cada uno contiene el chip DW1000, un procesador STM32F105 ARM Cortex M3 y una antena omnidireccional. Los nodos pueden ser configurados como anchor o como tag a través de un interruptor. Los nodos tipo anchor pueden medir la distancia a cada uno de los tags, y se comunican entre ellos para compartir los rangos medidos. Un dispositivo externo (por ejemplo un PC) puede conectarse a uno de los anchors por USB para recolectar los rangos entre cada tag y cada anchor.

Como configuración adicional de los nodos, es es posible elegir la frecuencia central de emisión (4 $\mathrm{GHz}$ o $6.5 \mathrm{GHz}$ ) y las tasas de transmisión de datos (110 kbps o $6.8 \mathrm{Mbps}$ ). Nosotros utilizamos la configuración por defecto (4 GHz y 110 kbps) que es la recomendada para operación a larga distancia. Una tasa de medida de rangos entre anchors y tags de $3.5 \mathrm{~Hz}$ es esperable según el fabricante.

\subsection{El equipo RTK-DGPS y el vehículo Autopia}

El vehículo empleado como plataforma de prueba cuenta con un receptor GNSS modelo Trimble BD960 con capacidad de realizar correcciones diferenciales en la medida desde estaciones de referencia y a través de alguno de los sistemas de aumentación disponibles por satélite (e.g. WAAS, EGNOS). En cada ciclo de medida, el receptor

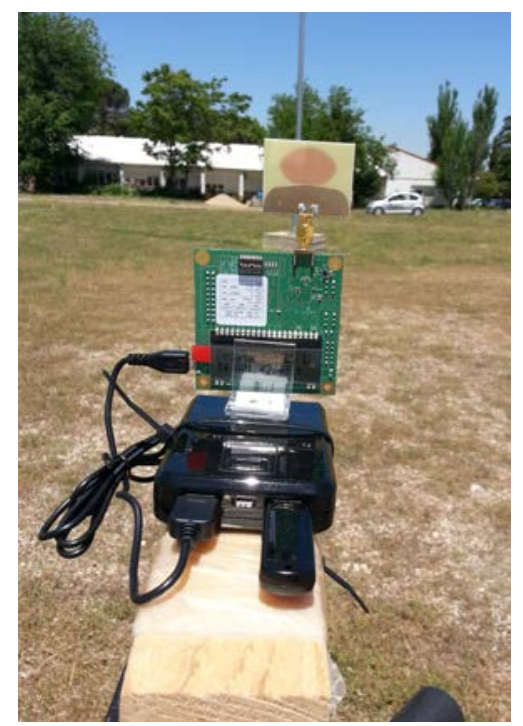

Figura 2: Detalle de uno de los nodos UWB de Decawave (tags) distribuidos por el entorno de test.

trasmite a través de una interfaz Ethernet un conjunto de mensajes codificados de acuerdo al protocolo NMEA-0183 [6]. En concreto, se emplean tres tipos de mensajes: (i) GPGGA, que contiene información del posicionamiento, incluyendo la calidad de la medida y el número de satélites utilizados; (ii) GPVTG, con información de la velocidad y orientación del vehículo; y (iii) GPGST, con las estadísticas de error para la medida recibida.

Dado que la motivación principal de este trabajo es la caracterización del sistema UWB para la localización de vehículos, el receptor se ha configurado para utilizar correcciones diferenciales desde una estación de referencia ubicada en las instalaciones del CAR [1]. De esta forma la medida del receptor tiene una precisión centimétrica.

\subsection{Distribución de nodos UWB}

De los 8 nodos UWB hemos configurado 6 como tags y 2 como anchors. Los 6 tags se colocan sobre unas bases verticales a 1.7 metros de altura y se distribuyen junto a las pistas privadas del centro CAR. Los dos anchors se colocan sobre la baca en el techo del coche separados entre sí una distancia $d_{1}$ de 0,83 metros y a una altura respecto al suelo de 1,7 metros. De esta forma tendremos 6 tags numerados como $T^{0}, T^{1}, . . T^{5}$ y 2 anchors numerados $A_{0}$ y $A_{1}$ colocados a la izquierda y derecha del vehículo tal y como se ve en la Figura 3.

Los tags en el entorno están en las posiciones $\left(T^{0}=[-93.63-39.83], \quad T^{1}=\left[\begin{array}{lll}-48.96 & -53.87\end{array}\right], \quad T^{2}=[-\right.$ $9.84-63.47], T^{3}=[-6.88-4.06], T^{4}=\left[\begin{array}{lll}-49.97 & -1.86\end{array}\right]$, $\left.T^{5}=\left[\begin{array}{ll}-90.64 & 1.78\end{array}\right]\right)$ en un sistema de referencia cuyo origen está en el punto global UTM [458965.88 


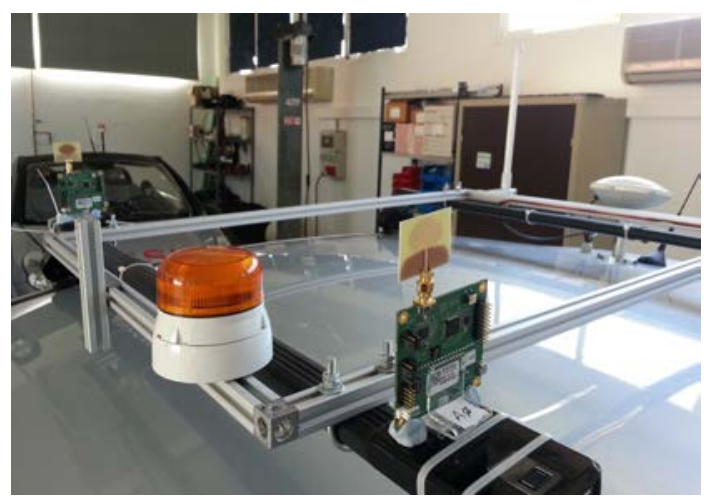

Figura 3: Detalle de la instalación de dos nodos UWB (anchors) sobre el frontal del techo del vehículo. Obsérvese la posición retrasada de la antena GPS.

4462547.73], estando los ejes X e Y orientados hacia el Este y Norte respectivamente.

Los anchors en el techo están adelantados una distancia $d_{2}$ de 1,05 metros sobre la ubicación de la antena GPS. Esta geometría se tendrá en cuenta posteriormente para poder estimar los errores de rango y de posición, así como para formular el filtro de Kalman de seguimiento del vehículo.

\subsection{Recorridos realizados con el vehículo}

Se han realizado 3 recorridos diferentes por las pistas de prueba (ver Figura 4). El recorrido 1 consiste en una trayectoria en forma de rectángulo incluida dentro del área cubierta por los 6 tags. El recorrido 2 es similar en cuanto a dimensiones pero más complejo ya que además incluye recorridos por una rotonda dentro de este recinto rectangular. El recorrido 3 realiza un ocho conformado por dos rectángulos, uno de ellos el mismo del recorrido 1 y otro rectángulo adicional ubicado al norte y fuera de la zona de despliegue de los 6 tags. En todos los casos la mayor distancia entre tag y anchor es de menos de 125 metros.

Los recorridos se han realizado a una velocidad lenta de unos $2.2 \mathrm{~m} / \mathrm{s}(8 \mathrm{~km} / \mathrm{h})$ a fin de evitar errores en la medida provocados por los retardos en la captura de datos UWB por el puerto serie, que pueden alcanzar hasta 0.4 segundos.

\section{Estudio de la precisión en la medida de rangos con UWB}

En esta sección queremos ver la precisión que conseguimos midiendo rangos con el equipo UWB. Como, gracias al GPS, conocemos con precisión la posición y la orientación del coche (salidas directas del sistema GPS), y además sabemos la posición exacta de los tags UWB (calibrados con
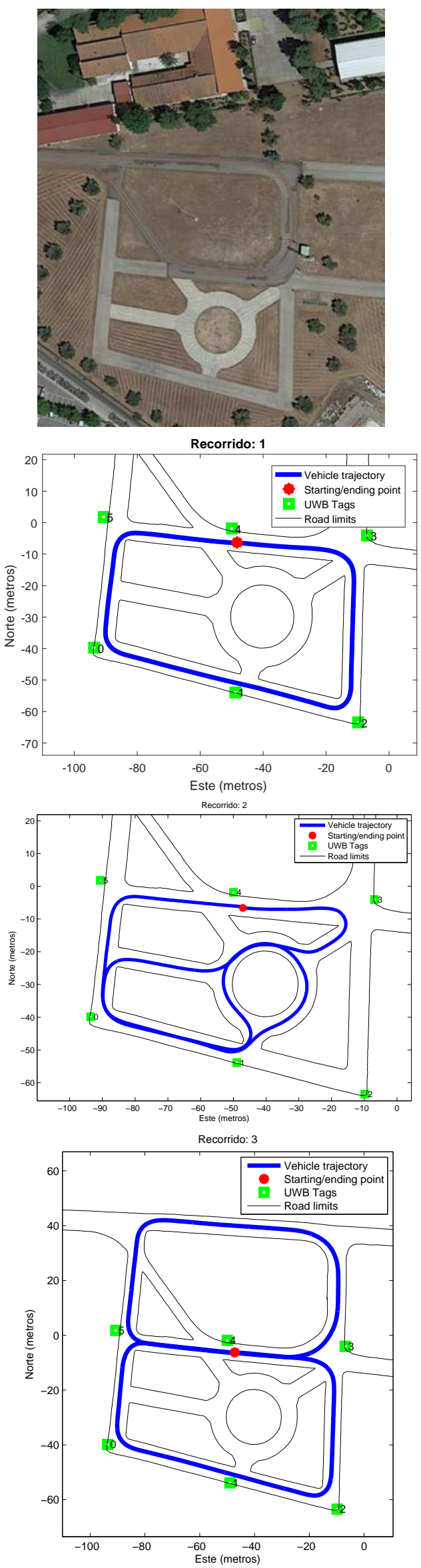

Figura 4: Pista de prueba del CAR, posición de balizas UWB y los 3 recorridos realizados. 
el mismo sistema GPS), entonces podemos calcular de forma exacta los rangos verdaderos que hay entre cada anchor $\left(A_{0}\right.$ y $\left.A_{1}\right)$ y los tags fijos UWB. Comparando estos rangos ideales con los rangos medidos experimentalmente podemos determinar el error de medida, que necesitamos caracterizar antes de intentar aplicar un algoritmo de localización.

La figura $5 \mathrm{a}$ y b muestra una superposición de los rangos medidos sobre los ideales, tanto para el $A_{0}$ como el $A_{1}$, en el caso del recorrido 1 . Se puede observar que hay bastantes intervalos temporales donde no es posible medir algunos de los rangos y que a partir de cierta distancia parece más difícil conseguir medir.

Si representamos la distancia real frente a la medida (Fig.5c) se observa claramente que existe una buena correspondencia y calidad de la medida, pero se ve también la existencia de ciertos valores con un error alejado de una distribución normal (outliers). Si exceptuamos esos outliers (que representan menos del $10 \%$ de las medidas) y nos concentramos en las mediciones cercanas a su valor verdadero (Fig.5d) vemos que su distribución es normal con una desviación estándar menor de 0.2 metros.

Para ver con mayor detalle el efecto de la distancia sobre la capacidad para medir rangos, presentamos en la figura 6 un histograma representando la probabilidad de detección. Se ve que a partir de 60 metros de distancia se detectan menos de los rangos que serían esperables a una tasa de medida constante.

Los resultados para los recorridos 2 y 3 son bastante parecidos en cuanto a conclusiones, es decir, una probabilidad de detección alta hasta 60 metros, aparición de diversos outliers, y una buena precisión para la mayoría de rangos.

\section{Método de posicionamiento propuesto}

En este apartado presentamos un algoritmo que utilizando los rangos UWB medidos permite estimar la posición del vehículo en el mismo punto que la antena $\operatorname{GPS}(x, y)$, así como su orientación $(\theta)$. Para ello utilizamos un único filtro de Kalman extendido (EKF) con 5 estados:

$$
\mathbf{X}=\left(x_{c}, y_{c}, v_{x}, v_{y}, \theta\right)
$$

donde $\left(x_{c}, y_{c}\right)=0,5\left(x_{0}+x_{1}, y_{0}+y_{1}\right)$ es la posición en el punto medio entre los dos anchors UWB en coordenadas Este-Norte (véase figura 7$),\left(v_{x}, v_{y}\right)$ es la velocidad del vehículo a lo largo de estos ejes
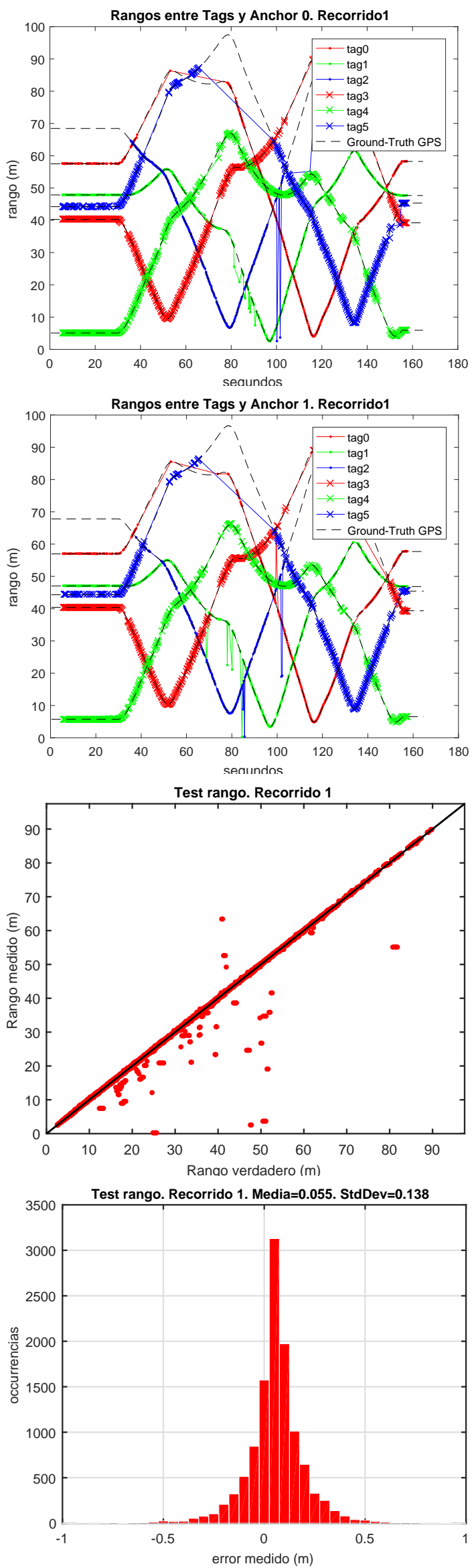

Figura 5: Rangos medidos con UWB vs rangos ideales a anchor $0 \mathrm{y}$ anchor 1 en recorrido 1 (a y b). Rango medido versus rango ideal para anchors 0 y 1 juntos (c) y distribución de error en rango si no se consideran los outliers. 


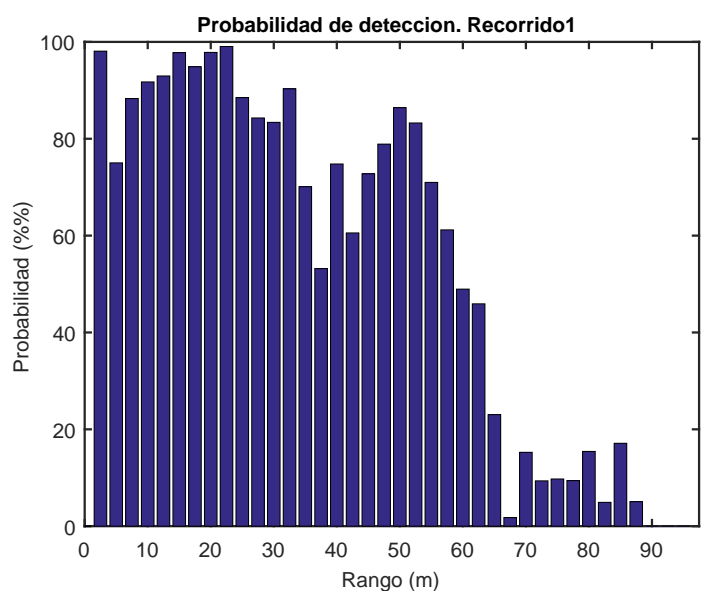

Figura 6: Probabilidad de detección y medida de un rango UWB según aumenta la distancia.

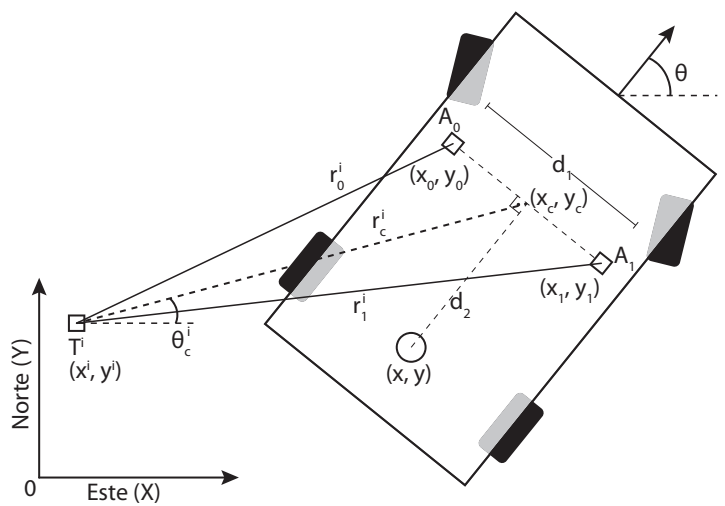

Figura 7: Esquema con la ubicación de los 2 anchors UWB $\left(A_{0}\right.$ y $\left.A_{1}\right)$ y la antena GPS sobre el techo del coche, y rangos medidos a un tag UWB cualquiera $T^{i}$.

Este-Norte, y $\theta$ la orientación del vehículo respecto al eje del Este (que no es exactamente igual al heading o dirección de avance).

La posición del centro de la antena GPS $(x, y)$, que se encuentra retrasada una distancia $d_{2}=1,05 \mathrm{~m}$, se obtendría, una vez estimada la orientación y la posición central de las antenas UWB, así:

$$
[x, y]=\left[x_{c}-d_{2} \cos (\theta), y_{c}-d_{2} \sin (\theta)\right]
$$

\subsection{Modelo de movimiento}

El modelo de movimiento utilizado es uno de velocidad constante con cambios de velocidad y giros del vehículo modelados como ruido de aceleración (a) y de velocidad de rotación $\left(v_{\theta}\right)$, estando estos últimos desacoplados (aproximación válida a bajas velocidades como en los experimentos realizados). Así:

$$
\mathbf{X}=f\left(x_{c}, y_{c}, v_{x}, v_{y}, \theta\right)+g_{a}(a)+g_{\theta}\left(v_{\theta}\right)
$$

El modelo en tiempo discreto sería:

$$
\mathbf{X}^{k+1}=\left(\begin{array}{c}
x_{c}^{k+1} \\
y_{c}^{k+1} \\
v_{x}^{k+1} \\
v_{y}^{k+1} \\
\theta^{k+1}
\end{array}\right)=\left(\begin{array}{c}
x_{c}^{k}+v_{x}^{k} d t+\frac{a^{k} d t^{2}}{2} \\
y_{c}^{k}+v_{y}^{k} d t+\frac{a^{k} d t^{2}}{2} \\
v_{x}^{k}+a^{k} d t \\
v_{y}^{k}+a^{k} d t \\
\theta^{k}+v_{\theta}^{k} d t
\end{array}\right)
$$

donde la matriz de transición de estados del filtro de Kalman es

$$
F=\left(\begin{array}{lllll}
1 & 0 & d t & 0 & 0 \\
0 & 1 & 0 & d t & 0 \\
0 & 0 & 1 & 0 & 0 \\
0 & 0 & 0 & 1 & 0 \\
0 & 0 & 0 & 0 & 1
\end{array}\right)
$$

$\mathrm{y}$ donde $G_{a}=\left[\frac{d t^{2}}{2}, \frac{d t^{2}}{2}, d t, d t, 0\right]^{\prime}$ y $G_{\theta}=$ $[0,0,0,0, d t]^{\prime}$.

Por tanto la matriz de covarianza del proceso o modelo de movimiento a utilizar en el EKF es:

$$
\begin{aligned}
Q= & G_{a} q_{a} G_{a}^{\prime}+G_{\theta} q_{\theta} G_{\theta}^{\prime}= \\
& \left(\begin{array}{lllll}
\frac{d t^{4}}{4} & 0 & \frac{d t^{3}}{2} & 0 & 0 \\
0 & \frac{d t^{4}}{4} & 0 & \frac{d t^{3}}{2} & 0 \\
\frac{d t^{3}}{2} & 0 & d t^{2} & 0 & 0 \\
0 & \frac{d t^{3}}{2} & 0 & d t^{2} & 0 \\
0 & 0 & 0 & 0 & q_{\theta} / q_{a}
\end{array}\right) q_{a}
\end{aligned}
$$

donde $q_{a}$ y $q_{\theta}$ son las covarianzas del ruido en aceleración y en rotación respectivamente.

El robustecimiento del filtro de Kalman para evitar los outliers más significativos se hace rechazando las actualizaciones del filtro cuando la innovación (discrepancia entre el rango predicho y el medido), medida como una distancia de Mahalanobis, es superior a un cierto umbral. De esta forma se eliminan buena parte de los outliers.

\subsection{Modelo de medida}

Las medidas disponibles son rangos que llegan a los 2 anchors de forma dispersa procedentes de diferentes tags, por tanto diferenciamos entre el rango del tag $T^{i}$ al anchor $A_{0}\left(r_{0}^{i}\right)$ y el rango del $\operatorname{tag} T^{i}$ al anchor $A_{1}\left(r_{1}^{i}\right)$. De esta forma el rango experimental medido $r_{j}^{i}$ entre anchor $j$ y tag $i$ dependerá de la posición del punto medio entre anchors $\left(x_{c}, y_{c}\right)$ y la rotación $\theta$ del vehículo de esta forma: 
$r_{j}^{i}=\sqrt{\left(x_{c}-x^{i}\right)^{2}+\left(y_{c}-y^{i}\right)^{2}}+s \cdot d_{1} / 2 \cdot \sin \left(\theta-\theta_{c}^{i}\right)$

donde la posición de los tags $T^{i}$ es $\left(x^{i}, y^{i}\right)(\mathrm{i}=0 . .5)$, el índice de los anchor $j$ puede valer 0 o $1, s$ es un signo que vale 1 si $\mathrm{j}=1$ y -1 si $\mathrm{j}=0, d_{1}$ es la distancia entre anchors $\left(d_{1}=0.83 \mathrm{~m}\right)$, y $\theta_{j}^{i}$ es el ángulo respecto al Este de la línea que pasa por el $\operatorname{tag} T^{i}$ y el centro de los anchors $\left(x_{c}, y_{c}\right)$; es decir: $\theta_{c}^{i}=\arctan \left(\frac{y_{c}-y^{i}}{x_{c}-x^{i}}\right)$.

Para obtener la matriz de observación $H$ del único filtro de Kalman utilizado, derivamos este modelo de medida (eq. 7) respecto a los términos del estado $\left(x_{c}, y_{c}, v_{x}, v_{y}, \theta\right)$, obteniendo:

$$
H=\left(\begin{array}{l}
\frac{x_{c}-x^{i}}{r_{c}^{i}}+\frac{s \cdot d_{1} / 2 \cdot \cos \left(\theta-\theta_{c}^{i}\right) \cdot\left(y_{c}-y^{i}\right)}{\left(r_{c}^{i}\right)^{2}} \\
\frac{y_{c}-y^{i}}{r_{c}^{i}}-\frac{s \cdot d_{1} / 2 \cdot \cos \left(\theta-\theta_{c}^{i}\right) \cdot\left(x_{c}-x^{i}\right)}{\left(r_{c}^{i}\right)^{2}} \\
0 \\
0 \\
s \cdot d_{1} / 2 \cdot \cos \left(\theta-\theta_{c}^{i}\right)
\end{array}\right)^{\prime}
$$

donde $H$ es una matriz de dimensión variable con tantas filas como rangos medidos y tantas columnas como los estados estimados $(5) ; r_{c}^{i}$ es el rango entre el tag $T^{i}$ y el centro de los anchors, es decir: $r_{c}^{i}=\sqrt{\left(x_{c}-x^{i}\right)^{2}+\left(y_{c}-y^{i}\right)^{2}}$.

\section{Estudio de la precición en la estimación de la posición y orientación del vehículo}

En esta sección vamos a mostrar la calidad de las estimaciones de posicionamiento y de orientación que hemos podido obtener usando las medidas de los dispositivos UWB y el filtro de Kalman extendido descrito en la sección anterior.

En las siguientes figuras (Fig.8, Fig.9, Fig.10) podemos ver los resultados obtenidos para los 3 recorridos de test. En la parte de arriba de las figuras (a) vemos la trayectoria estimada con UWB superpuesta a la posición verdadera proporcionada por el GPS-RTK. A pesar de que la disponibilidad de rangos no es muy alta (típicamente entre solo entre 3 y 4 tags son visibles de los 6 instalados) y de que existen algunos outliers con varios metros de error, el filtro de Kalman permite estimar con relativa precisión la posición.

El error de posición temporal, según avanza el vehículo se ve en la segunda gráfica de cada figura (b). Existen momentos donde solo están disponibles 2 o menos tags, lo cual provoca una falta de información que limita las estimaciones, ya que
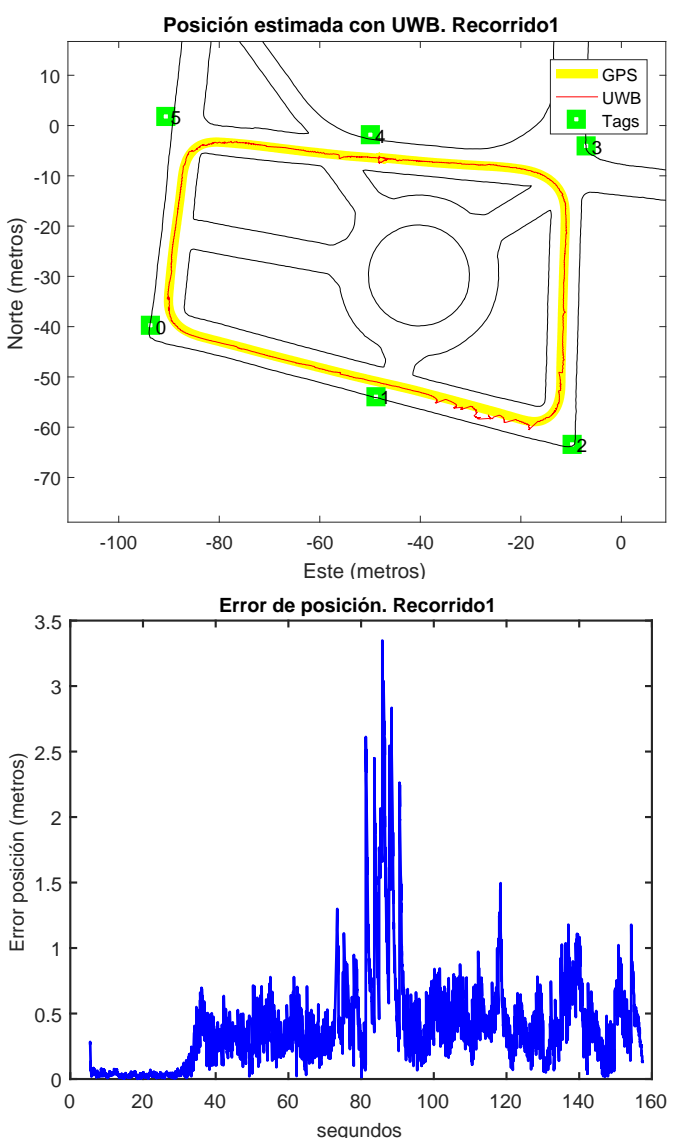

CDF. Error medio: 0.402; Error RMS: 0.562; Error 90\%: 0.738
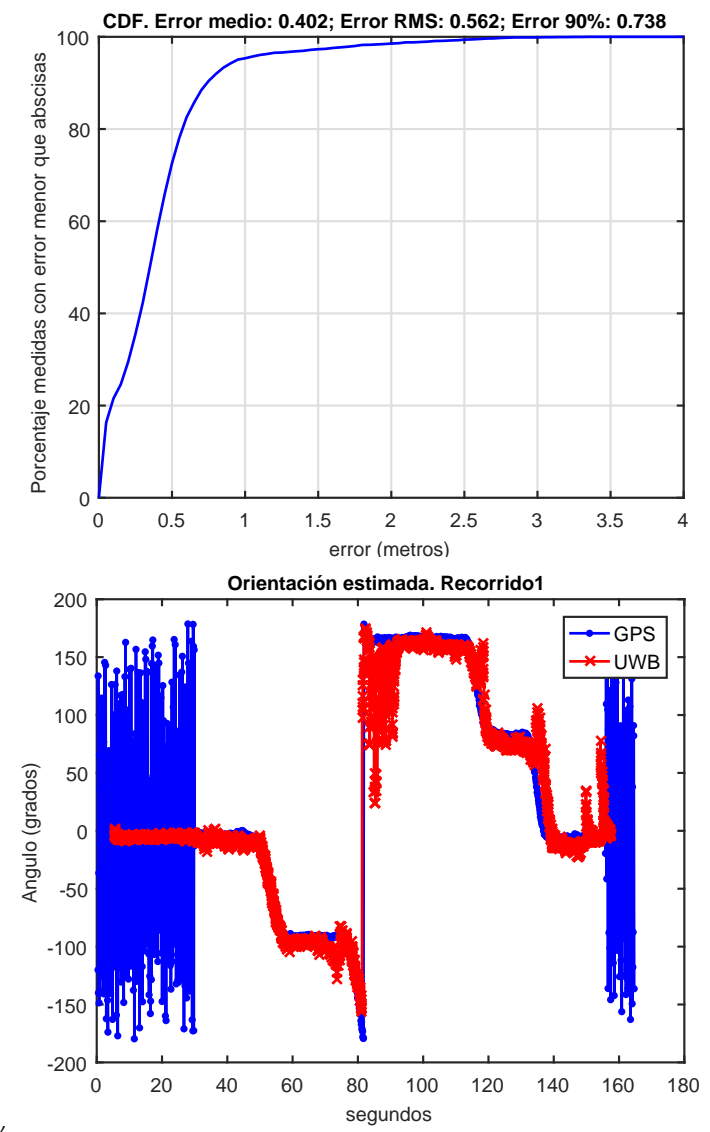

Figura 8: Posición estimada en recorrido 1 sobre un mapa (a), error de posición (b), CDF del error en posición (c), y estimación de orientación (d). 
a)

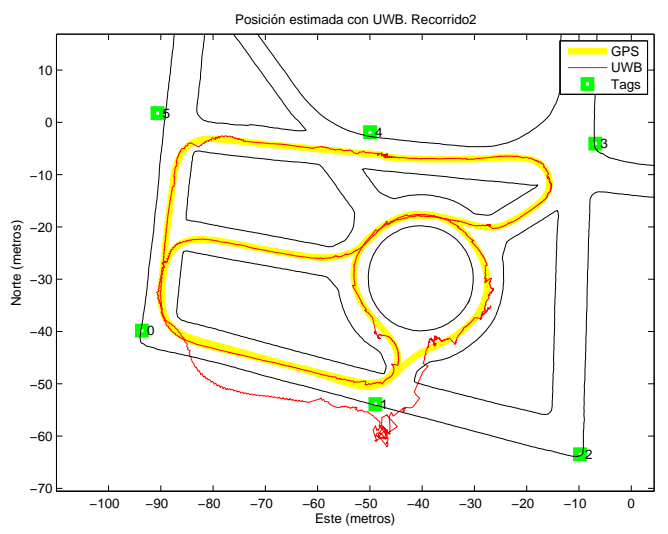

b)

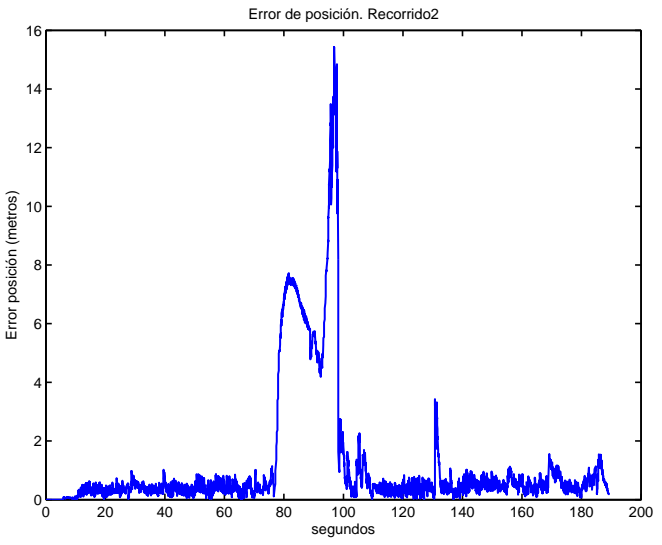

c)
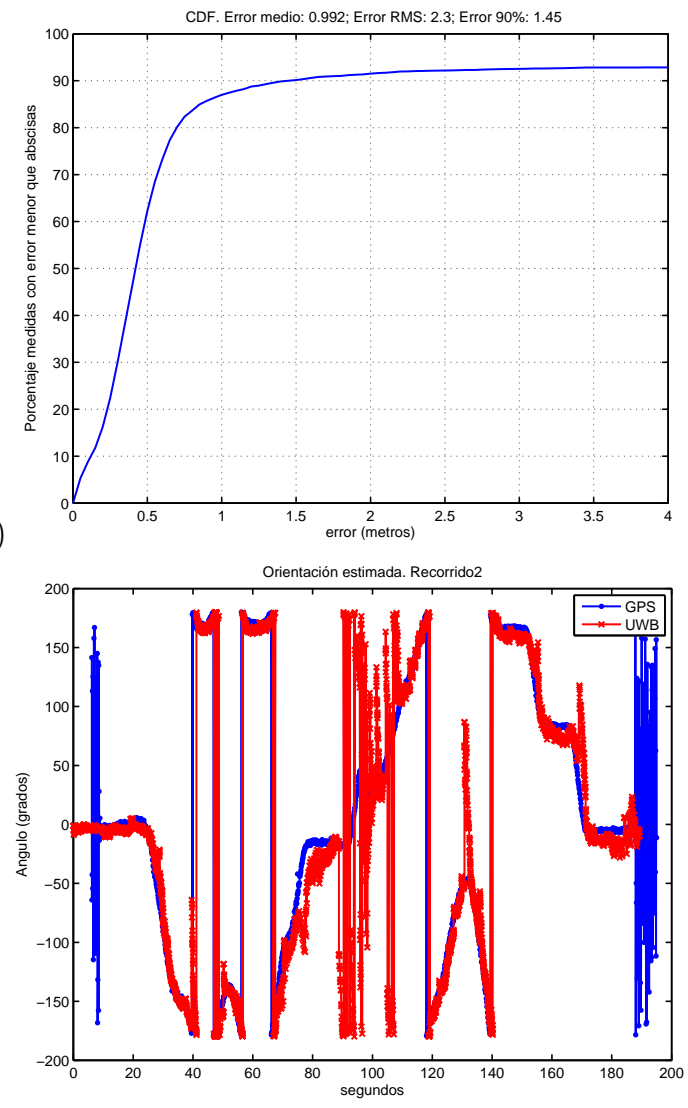

d)

Figura 9: Posición estimada en recorrido 2 sobre un mapa (a), error de posición (b), CDF del error en posición (c), y estimación de orientación (d).

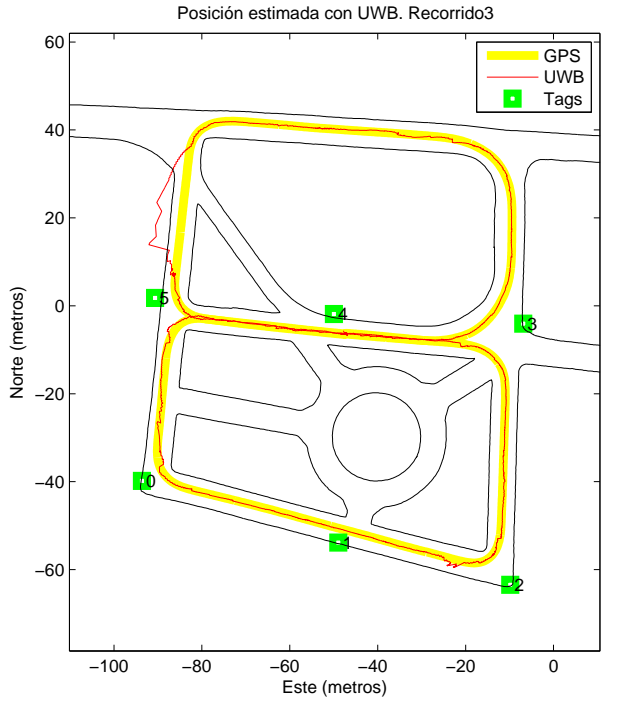

a)

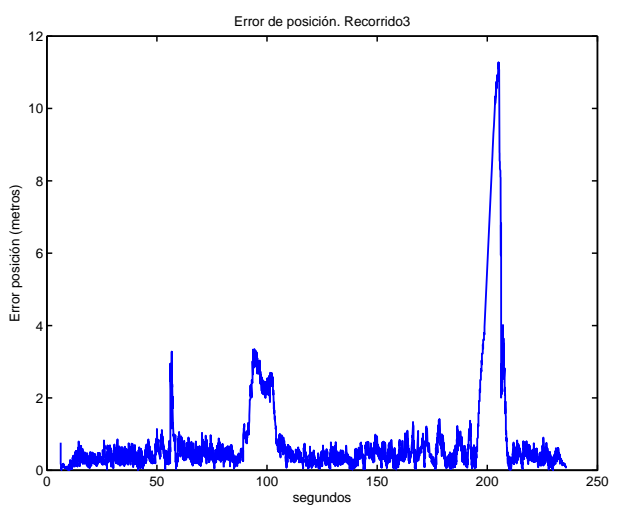

b)

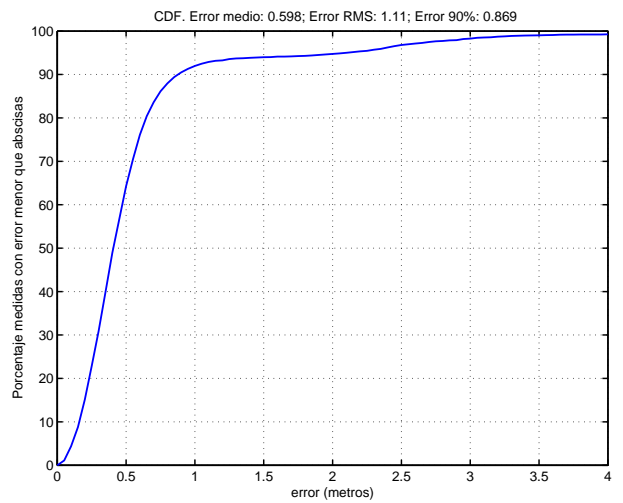

c)

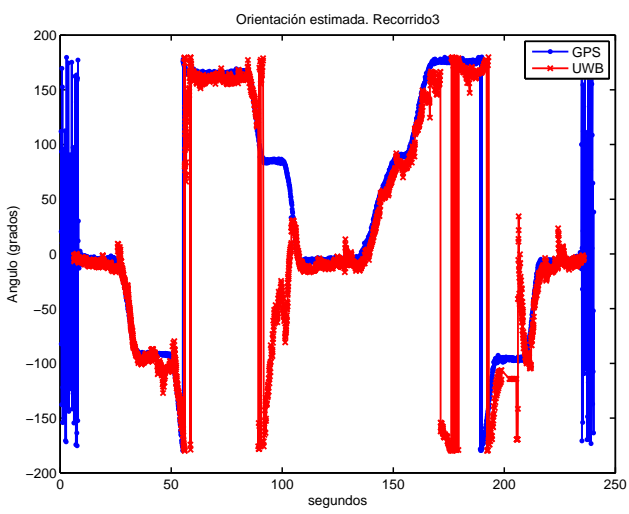

Figura 10: Posición estimada en recorrido 3 sobre un mapa (a), error de posición (b), CDF del error en posición (c), y estimación de orientación (d). 
aumentan de incertidumbre al basarse la salida del filtro de Kalman en una predicción de los estados sin apenas actualizaciones con medidas de rango. El error medio de posición en todos los recorridos es de 0.4, 0.99 y 0.60 metros respectivamente. Siendo el error RMS de 0.73, 1.45 y 1.11 metros, respectivamente. La distribución de este error integrada se puede ver en la gráficas (c) de las figuras como una distribución acumulativa del error (CDF -Cumulative Distribution Function).

Finalmente la estimación de la orientación del vehículo se muestra en las figuras 8 a 10 en la gráfica inferior (d), junto con el heading, o ángulo en la dirección de avance, generado por el GPS. Se aprecia que al principio del recorrido con el coche parado el heading del GPS es totalmente ruidoso (ya que no hay movimiento y no se puede estimar hacia dónde avanza el coche). Sin embargo la estimación de orientación basada en UWB es bastante correcta (aspecto que se ve claro en la Fig.8d). Durante la fase de movimiento del vehículo la orientación es bastante similar al heading (ya que el coche se mueve siempre hacia delante), salvo en las zonas donde la falta de suficientes medidas correctas de rango es patente, que afecta tanto a la estimación de posición como a la de orientación.

\section{Conclusiones}

En este trabajo se ha presentado una evaluación experimental de un sistema de radio UWB para localizar vehículos en entornos donde el posicionamiento con GPS pueda no estar disponible o esté degradado. Hemos realizado un análisis a nivel de medida de rangos de la capacidad de medida entre pares de nodos UWB, y hemos visto que si bien existen medidas erróneas (outliers) y un rango de operación limitado a partir de 60 metros, es posible medir en general con precisiones submétricas (desviación estándar menor de 0.2 metros) la distancia entre nodos. Planteando un filtro de Kalman extendido para obtener la posición y orientación del vehículo ha sido posible determinar con bastante precisión dichas estimaciones (errores de posición menores a 0.7 metros en el $90 \%$ de los casos) eliminando gran parte de los outliers. Los mayores errores de posición y orientación han ocurrido cuando solo teníamos 2 o menos tags UWB a la vista. En estos casos la trilateración no es posible por falta de información sensorial y es normal que el filtro diverja al usar solo predicciones basadas en los estados previos.

Como trabajo futuro está previsto analizar el efecto de subir ligeramente la posición de los anchors UWB sobre el techo del vehículo o aumentar la altura de las bases de los anchors, para de esta forma ver si se maximizaría el rango de medida y la pro- babilidad de detección. Un importante trabajo futuro consiste en extender el uso del sistema UWB al posicionamiento relativo entre vehículos, con el fin de evaluar la aplicabilidad de estos sistemas en la gestión de maniobras cooperativas, como es el caso de la formación de pelotones (platooning). Así mismo, se plantea aprovechar la información de sensores adicionales de la plataforma de prueba (odometría y unidades inerciales) para mejorar el filtrado de la localización y evaluar el desempeño en entornos donde la localización por GPS es inviable, como es el caso de túneles y zonas subterráneas.

\section{Agradecimientos}

Los autores quieren agradecer la ayuda financiera recibida de los proyectos LORIS (TIN201238080-C04-04), TARSIUS (TIN2015-71564-C4-2R (MINECO/FEDER)), TCAP-AUTO (RTC2015-3942-4 (MINECO/FEDER)) y NAVEGASE (DPI2014-53525-C3-3-R (MINECO)).

\section{Referencias}

[1] J. Godoy, V. Milanés, J. Rastelli, J. Villagrá, T. de Pedro, C. González. Implementación de un sistema de localización para vehículos sin conductor, Seminario Anual de Automática, Electrónica Industrial e Intrumentación 2010.

[2] I. Guvenc, C. C. Chong, F. Watanabe, "NLOS identification and mitigation for UWB localization systems," IEEE Wireless Communications and Networking Conference, WCNC, 2007, pp. 1573-1578.

[3] A.R. Jiménez, F. Seco, C. Prieto, J. Roa, Tecnologías sensoriales de localización para entornos inteligentes, I Congreso español de informática-Simposio de Computación Ubicua e Inteligencia Ambiental, UCAmI2005, 2005, pp. $75-86$.

[4] E. Kaplan, C. Hegarty. Understanding GPS: Principles and applications. Artech House 1996.

[5] R. Mautz, Indoor Positioning Technologies. ETH Zurich, PhD thesis, 2012.

[6] National Marine Electronics Association (U.S.). Standard for Interfacing Marine Electronic Devices 2002.

[7] F. G. H. Nurminen, T. Ardeshiri, R. Piche, "A NLOS-robust TOA positioning filter based on a skew- t measurement noise model," in International Conference on Indoor Positioning and Indoor Navigation (IPIN), 2015, no. October, pp. 13-16. 